\title{
Early Tracking Behavior in Small-field Quintessence Models
}

\author{
Wei Wang ${ }^{1}$, Bo Feng ${ }^{2}$ \\ ${ }^{1}$ National Astronomical Observatories, Chinese Academy of Sciences, Beijing 100012, China, \\ wwang@lamost.bao.ac.cn \\ ${ }^{2}$ Institute of High Energy Physics, Chinese Academy of Sciences, P.O. Box 918-4, Beijing \\ 100049, China,fengbo@mail.ihep.ac.cn
}

\begin{abstract}
We study several quintessence models which are singular at $Q=0$, and use a simple initial constraint $Q_{i} \geq H_{\text {inflation }} / 2 \pi$ to see when they enter tracking regime, disregarding the details of inflation. We find it can give strong constraints for the inverse power-law potential $V=V_{0} Q^{-\alpha}$, which has to enter tracking regime for $\ln z \sim$ 10. While for the supergravity model $V=V_{0} Q^{-\alpha} \exp \left(k Q^{2} / 2\right)$, the constraint is much weakened. For another kind inverse power-law potential $V=V_{0} \exp (\lambda / Q)$, it exhibits no constraints.
\end{abstract}

Subject headings: quintessence, tracking

\section{Introduction}

Recent observations by type Ia supernova(SN) survey(Garnavich et al. 1998, Perlmutter et al. 1998) and cosmic microwave background(CMB) anisotropies(de bernardis et al. 2002, Lee et al. 2001) strongly show the evidence for a cosmological constant or dark energy. In general case, the dark energy (or Quintessence) can have a time-dependent equation of state, $P_{Q}=\omega_{Q}(t) \nu_{Q}$ (Wetterich 1988, Peebles \& Ratra 1988), which is invoked to explain the coincidence problem. Some researchers have reanalyzed the cosmological data from CMB, SN, large scale structure(LSS) and gravitational lens statistics(Bean \& Melchiorri 2001, Baccigalupi et al. 2001, Hannestad \& Mörtsell, 2002, Chae et al. 2002), confirming that quintessence is slightly preferred with respect to cosmological constant.

An important class of quintessence models are known as tracking models. By coupling a scalar field to matter one can obtain tracking solutions (Zlatev et al. 1998, Steinhardt et al. 1999) for time dependence of dark energy density so that it always follows the dominant energy density component and fairly independent of initial conditions. Recently Malquarti \& Liddle(2002) used stochastic inflation formalism to constrain the initial values of quintessence after inflation. They have shown that for inverse power-law form of quintessence $V=V_{0} Q^{-\alpha}$ satisfying current observations, initial $Q$ was so large that it could not enter tracking regime until the matter-domination epoch. This has put the tracking behavior in considerable jeopardy for such quintessence models. 
In the present paper, we will study the behaviors of several quintessence models which are singular at $Q=0$ (dubbed "Small-field Quintessence Models") affected by inflation. Quintessence being almost massless, one has $\delta Q \sim H / 2 \pi$ (See Liddle et al 1993 for details ) after inflation. The simplest constraint on initial $Q$ is that its value should be larger than the perturbative part: $Q \geq \delta Q$, i.e. $Q \geq H / 2 \pi$. We shall use this constraint to see when they enter tracking regime, disregarding the details of inflation. In the following section, we will discuss the tracking behaviors of three quintessence models: inverse power-law potential $V=V_{0} Q^{-\alpha}$ (Ratra \& Peebles 1988), the supergravity model $V=V_{0} Q^{-\alpha} \exp \left(k Q^{2} / 2\right)$ and the exponential form of inverse power-law potential $V=V_{0} \exp (\lambda / Q)$.

\section{Models and tracking solutions}

We shall consider models of quintessence in a flat cosmological background, i.e. $\Omega_{k}=0$. The ratio of energy density to the critical density today is $\Omega_{Q}$ for the $Q$-field and $\Omega_{m}$ for the matter density where $\Omega_{m}+\Omega_{Q}=1$. We also define a background equation-of-state $\omega_{B}, \omega_{B}=1 / 3$ in radiation-dominated epoch and 0 in matter-dominated era. We use dimensionless units where the Planck mass is $\mathrm{M}_{p l}=1$.

The equation of motion for the $Q$-field is

$$
\ddot{Q}+3 H \dot{Q}+V^{\prime}=0
$$

where $V^{\prime}=\ddot{Q} \frac{1-\omega_{Q}}{1+\omega_{Q}}$ and

$$
H^{2}=\left(\frac{\dot{a}}{a}\right)^{2}=\frac{8 \pi G}{3}\left(\rho_{Q}+\rho_{B}\right),
$$

$a$ is the Robertson-Walker scale factor, $\rho_{B}=\rho_{m}+\rho_{r}, \rho_{m}$ and $\rho_{r}$ are the matter and radiation energy density respectively. Early in the radiation-dominated epoch, we have $H \approx 1 / 2 t$ and substituting the form of $V^{\prime}$ into Eq.(1):

$$
\ddot{Q}+\frac{3\left(1+\omega_{Q}\right)}{4 t} \dot{Q}=0
$$

Assuming $\omega_{Q}$ to be a constant, we can easily obtain the solution of the equation: $\dot{Q}=C t^{-3\left(1+\omega_{Q}\right) / 4}$, where $\mathrm{C}$ is a constant. So we have

$$
\Omega_{Q}=\left(\frac{\dot{Q}}{H}\right)^{2} \propto t^{-\frac{3}{2}\left(1+\omega_{Q}\right)+2} \propto a^{-3\left(1+\omega_{Q}\right)+4} .
$$

The above equation gives the rough evolution of $Q$-field. On the other hand we can obtain the $Q$ energy density in another method. According to the present cosmological density $\rho_{0}$, we can write

$$
\rho_{Q}=\rho_{0}\left(\frac{a_{\mathrm{eq}}}{a_{0}}\right)^{-3\left(1+\omega_{1}\right)}\left(\frac{a}{a_{\mathrm{eq}}}\right)^{-3\left(1+\omega_{2}\right)}
$$

or

$$
\ln \rho_{Q}=\ln \rho_{0}+3\left(\omega_{1}-\omega_{2}\right) \ln \left(z_{\mathrm{eq}}+1\right)+3\left(1+\omega_{2}\right) \ln (z+1),
$$


where $z$ is the redshift, the subscript eq denotes the epoch of matter-radiation equality, and $\omega_{1}$ and $\omega_{2}$ are the equations of state of Q-field during matter- and radiation-domination epochs. Note in the analytical formula Eqs. $(5,6)$ we've assumed $\omega_{1}$ to be a constant and today is matter-dominant, this has led to considerably uncertainties when compared to the exact numerical case (See Fig.3).

An important function is $\Gamma \equiv V^{\prime \prime} V /\left(V^{\prime}\right)^{2}$, whose properties determine whether tracking solutions exist. Taking the derivative of the equation-of-motion with respect to $Q$ and combining with the equation-of-motion itself, we can obtain the tracking equation:

$$
\Gamma \equiv 1+\frac{\omega_{B}-\omega_{Q}}{2\left(1+\omega_{Q}\right)}-\frac{1+\omega_{B}-2 \omega_{Q}}{2\left(1+\omega_{Q}\right)} \frac{\dot{x}}{6+x}-\frac{2}{\left(1+\omega_{Q}\right)} \frac{\ddot{x}}{(6+\dot{x})^{2}},
$$

where $x \equiv\left(1+\omega_{Q}\right) /\left(1-\omega_{Q}\right), \dot{x} \equiv d \ln x / d \ln a$ and $\ddot{x} \equiv d^{2} \ln x / d \ln a^{2}$.

In the following sections, we will discuss the tracking behavior of different quintessence models in detail. And we take the cosmological parameters derived from recent observational constraints throughout, $\Omega_{m}=0.3, \omega_{Q}=-0.82$ and the Hubble constant $h=0.65$.

\subsection{Pure Inverse power-law models}

The quintessence models of the pure inverse power-law potentials, as originally introduced by Ratra \& Peebles (1988): $V=V_{0} Q^{-\alpha}$. For the tracking solution,

$$
\Gamma-1=\frac{\omega_{B}-\omega_{Q}}{2\left(1+\omega_{Q}\right)}=\frac{1}{\alpha}
$$

then $\omega_{Q}=\alpha \omega_{Q}-2 /(\alpha+2)$. Since $\omega_{1}=-2 /(\alpha+2)\left(\omega_{B}=0\right)$ and $\omega_{2}=\alpha-6 /(3 \alpha+6)\left(\omega_{B}=1 / 3\right)$, according to Eq. (6), we have

$$
\ln \rho_{Q}=\ln \rho_{0}-\frac{\alpha}{\alpha+2} \ln \left(z_{\mathrm{eq}}+1\right)+\frac{4 \alpha+12}{\alpha+2} \ln (z+1) .
$$

For $\omega_{Q}=\rho_{Q}-2 V / \rho_{Q}$, we then have $V_{0} Q^{-\alpha}=V=\rho_{Q}\left(1-\omega_{Q}\right) / 2$, i.e. $\ln V_{0}-\alpha \ln Q=\ln \frac{1-\omega_{Q}}{2} \rho_{Q}$. Taking the approximation $V_{0} \simeq \rho_{0}$, we can obtain the final analytical form:

$$
\ln Q=\left(\ln \rho_{0}-\ln \frac{1-\omega_{Q}}{2} \rho_{Q}\right) / \alpha
$$

We have also computed the tracking behavior of the quintessence models numerically according to Eqs. (1) and (2). In Fig. 1, the dashed $(\alpha=1.4)$ and dotted $(\alpha=0.67)$ lines show the evolution of $Q$-field with the different values of $\alpha$. We have $Q \propto(z+1) * \operatorname{Exp}(-(4 \alpha+12) / \alpha=/(\alpha=+2))$ and $Q \sim 1$ today. For a smaller $\alpha, Q$ would be smaller earlier in the tracking regime, as shown in Fig.1. Our fit with $\Omega_{m}=0.3, \omega_{Q}=-0.82$ requires $\alpha \approx 0.67$ and $V_{0}^{1 / 4}=1.8 * 10^{-31}$. In Malquarti \& Liddle(2002), their fitting gives $0 \leq \alpha \leq 1(68 \%$ confidence), our result is in agreement with theirs. 


\subsection{Supergravity models}

In this section we consider the supergravity version of model considered previously with a superpotential of the form $V \propto Q^{-\alpha}$. We take the potential form $V=V_{0} Q^{-\alpha} \exp \left(k Q^{2} / 2\right)(\operatorname{Brax}$ et al 2000), where $k=8 \Pi G$, and study its tracking behavior.

For tracking solutions, we have

$$
\Gamma-1=\frac{\left(k+\alpha Q^{-2}\right) V^{2}+\left(k Q-\alpha Q^{-1}\right)^{2} V^{2}}{\left(k Q-\alpha Q^{-1}\right)^{2} V^{2}}-1=\frac{k+\alpha Q^{-2}}{\left(k Q-\alpha Q^{-1}\right)^{2}} .
$$

Because $k \ll \alpha Q^{-2}(Q \rightarrow 0), \Gamma-1 \simeq 1 / \alpha$. It is similar with former inverse power-law potentials. For $\omega_{Q}=-0.82$, and $\Omega_{m}=0.3, \alpha=11$ and $V_{0}^{1 / 4}=1.93 \times 10^{-32}$ are expected.

In Fig. 1, the solid line shows the $Q$-field evolution with the redshift. And in Fig. 2, we also show the evolution of the equation-of-state $\omega_{Q}$ in both the inverse power-law and the supergravity potentials, taking the same parameter $\alpha=11$. In the early radiation-dominant epoch, they have the same equation of state, while later when matter and quintessence dominate, $Q$ grows larger and the factor $\exp \left(k Q^{2} / 2\right)$ takes effect today in the supergravity model, which makes the main contribution to the different behaviors of the two models today. The pure inverse power law form $V=V_{0} Q^{-11}$ is ruled out for it predicts $\omega_{Q}>-0.3$ and cannot give an accelerating universe today, meanwhile $V=V_{0} Q^{-\alpha} \exp \left(k Q^{2} / 2\right)$ is still not excluded. In the two models, we have given the analytical forms for the tracking behavior according the Eqs. (9) and (10); we have also compared the results of analytical and numerical calculations in the supergravity version in Fig.3.

\subsection{Exponential Form of inverse power-law model}

Here, we further consider another kind of inverse power-law potential with the form like $V=$ $V_{0} e^{\lambda / Q}$. In our case, $\lambda \approx 0.3$ and $V_{0}^{1 / 4}=1.85 \times 10^{-31}$. However, since the equation-of-state of the model $\omega_{Q}$ varies with $t$, it is relatively difficult to obtain the analytical solutions. In Fig. 4 we have shown the evolution of the $Q$-field and the equation-of-state $\omega_{Q}$ by numerical calculations, the tracking behavior is different from the previous two models discussed above. The evolution curve of $Q$-field always rises with time. We are able to give some rough estimations since $Q<<1$ is also satisfied for large $z$. The form $e^{\lambda / Q}$ can be expanded to $Q^{-\alpha}$ series with $\alpha \rightarrow \infty$, hence $\omega_{Q}=(\alpha-6) /(3 \alpha+6) \approx 1 / 3$. Its early behavior of $Q$ and $\rho_{Q}$ can also be explained as $\alpha \rightarrow \infty$, where $Q$ would be much larger than in the pure inverse power-law models from above analysis.

For the comparison of the three models, we consider the very early behavior of $Q$-field. In the very early time, the three models take the same form $V=V_{0} Q^{-\alpha}$, with $\alpha=0.67,11$ and $\infty$, respectively.

In Fig. 5, we show our constraints on the tracking behavior of the quintessence models by taking the initial condition $Q_{i}=H / 2 \pi$, where $H \sim 10^{-5}$. We take $\dot{Q}_{i}=0$ initially. We do not 
include the exponential form of inverse power-law model because we have no constraints on it, as can be seen from Fig.4. The solid line displays the evolution of $Q$-field in the supergravity model, while the dashed line denotes the inverse power-law model. For $V=V_{0} Q^{-\alpha}$, it can only enter tracking regime after $\ln z \sim 10$. For the supergravity model $V=V_{0} Q^{-\alpha} \exp \left(k Q^{2} / 2\right)$, it requires $\ln (z+1) \leq 43$.

\section{Conclusions and Discussions}

We have analyzed the dynamical evolution and tracking solutions of three quintessence models. We used a simple constraint $Q_{i} \geq H / 2 \pi$ to study the tracking behaviors, and found that it can also give a strong constraint on the pure inverse power-law model which enters tracking regime at a late stage $\ln z \sim 10$. The key fact is that for such a pure inverse power-law model, $\alpha$ has to be very small in order to fit current observations. While for the supergravity model and exponential form of inverse power-law model, the exponential form takes a positive effect, rendering them have $\omega_{Q} \sim-1$ today and satisfy the CMB and SN constraints, meanwhile they take the pure inverse power-law form with much larger $\alpha$ and hence little constraint is exhibited with $Q_{i} \geq H / 2 \pi$ when entering the tracking regime.

The typical tracking behavior of inverse power-law model begins only at quite a late stage of evolution, well after nucleosynthesis and possibly after decoupling too as presented by this paper and Malquarti \& Liddle. Tracking is the key to solve the coincidence problem, tracking quintessence would lose the significance if it has to enter tracking regime extremely late. Therefore, the supergravity and exponential form of inverse power law model models show better tracking behaviors in our analysis.

We are grateful to Mingzhe Li, Xiulian Wang, De-Hai Zhang, Xinmin Zhang and Yongheng

Zhao for helpful discussions. This work is supported by National Natural Science Foundation of China under grant 10273011.

\section{REFERENCES}

Bean, R. \& Melchiorri, A. 2001, astro-ph/0110472

Baccigalupi, C. et al. 2001, astro-ph/0109097

Chae, K.H. et al. 2002, astro-ph/0209602

de Bernardis, P. et al. 2002, ApJ, 564, 559

Brax P., Martin J., 2000, Phys. Rev. D61, 103502

Garnavich, P.M. et al. 1998, ApJ, 493, L53

Hannestad, S. \& Mörtsell, E. 2002, astro-ph/0205096 
Lee, A.T. et al. 2001, ApJ, 561, L1

Liddle A. R., Lyth D. H., 1993, Phys. Rept., 231, 1

Malquarti, M. \& Liddle, A.R. 2002, astro-ph/0203232

Peebles, P.J.E. \& Ratra, B. 1988, ApJ, 325, L17

Permutter, S. et al. 1998, Nature, 391, 51

Ratra, B. \& Peebles, P.J.E. 1988, Phys. Rev. D37, 3406

Steinhardt P. J., Wang L.-M., Zlatev I., 1999, Phys. Rev. D59, 123504

Wetterich, C. 1988, Nucl. Phys. B302, 668

Zlatev I., Wang L.-M., Steinhardt P. J., 1999, Phys. Rev. Lett. 82, 896 


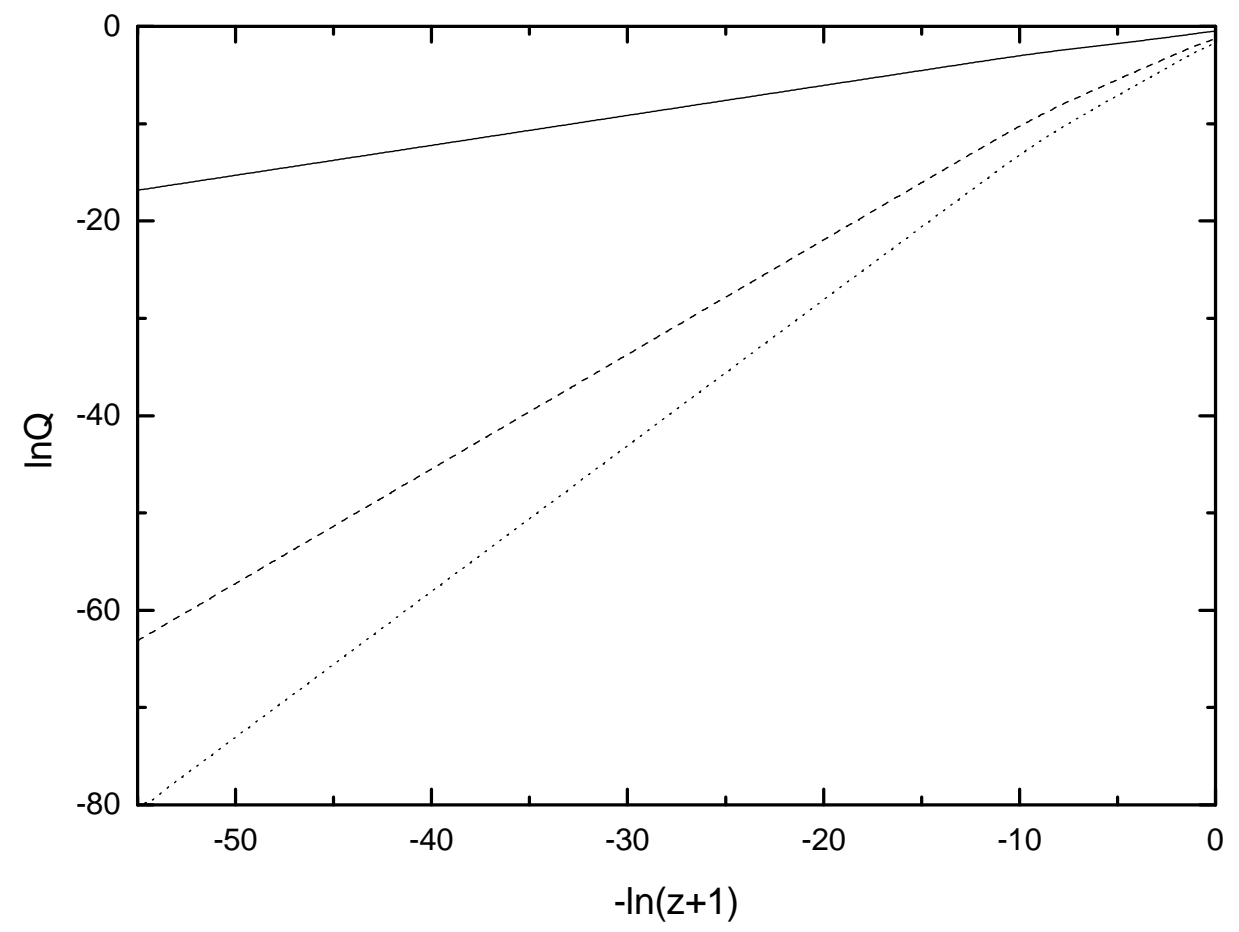

Fig. 1. - The evolution of the $Q$-field with the redshift. The solid line denotes the supergravity model, and the dashed and dotted lines display inverse power-law models with $\alpha=1.4$ and $\alpha=0.67$ respectively. 


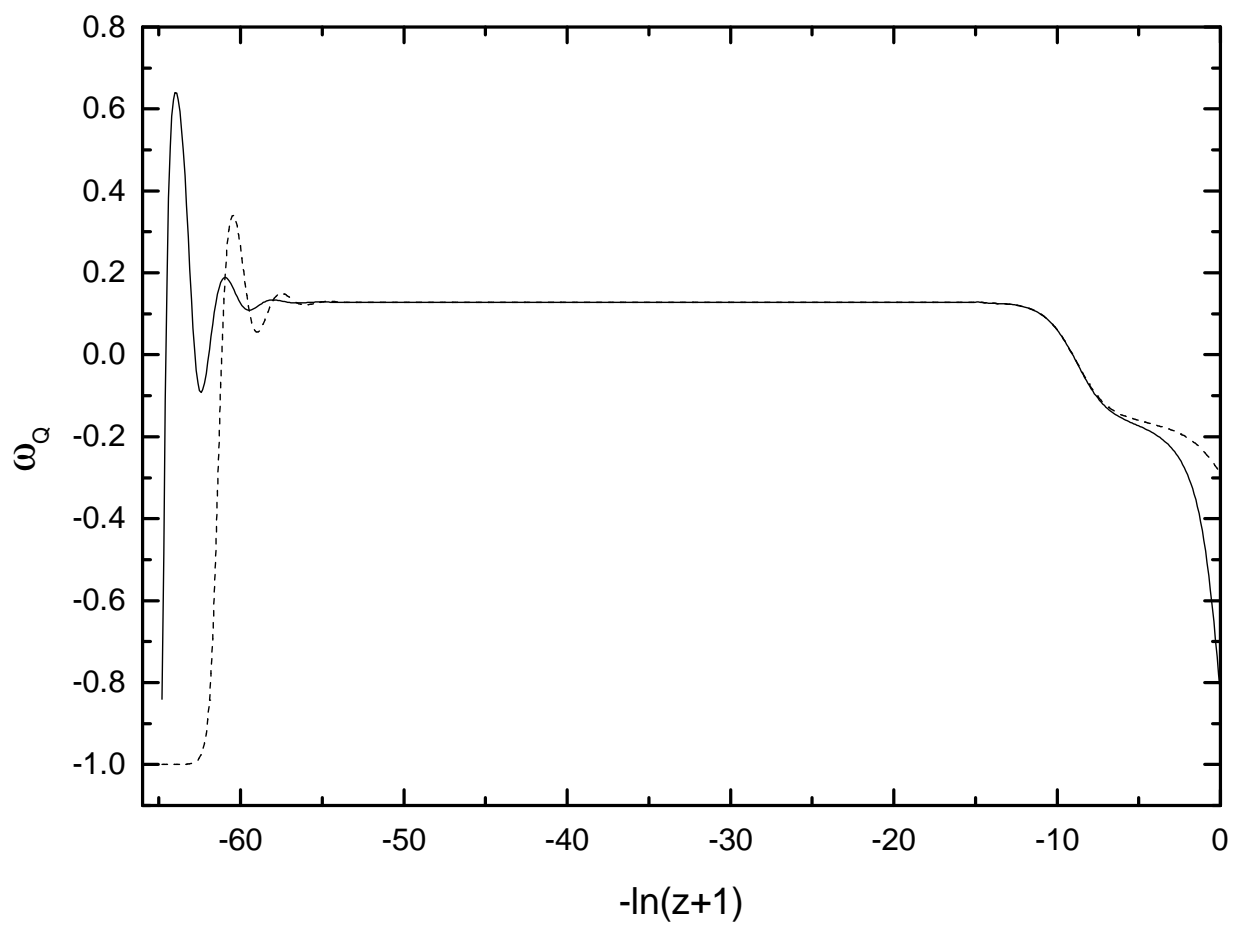

Fig. 2. - The different tracking behaviors between the inverse power-law model(dashed) and the supergravity model(solid), where $\alpha=11$. 

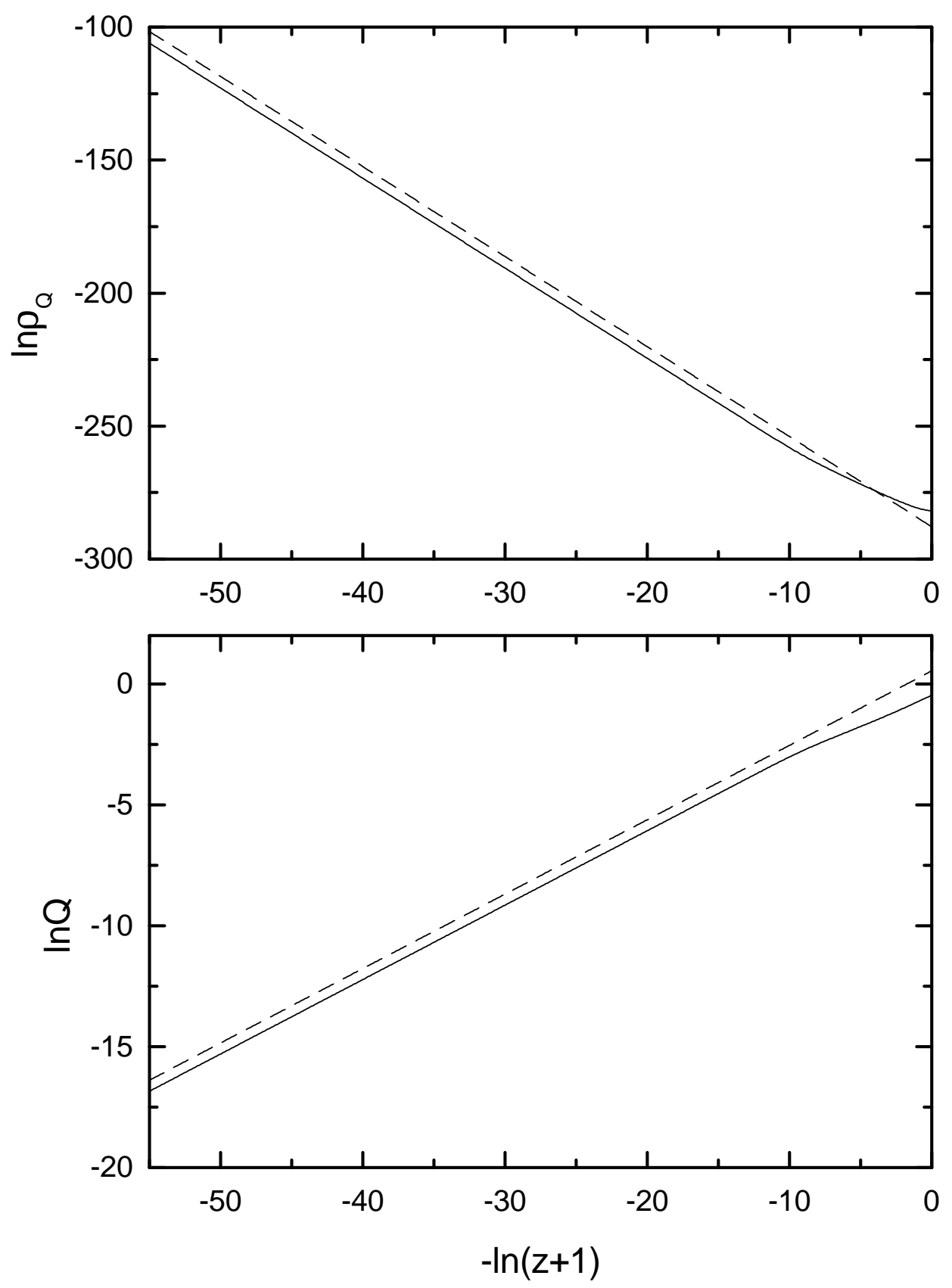

Fig. 3.- Comparison between the analytical(solid) and numerical(dashed) tracking solutions in the supergravity model. 

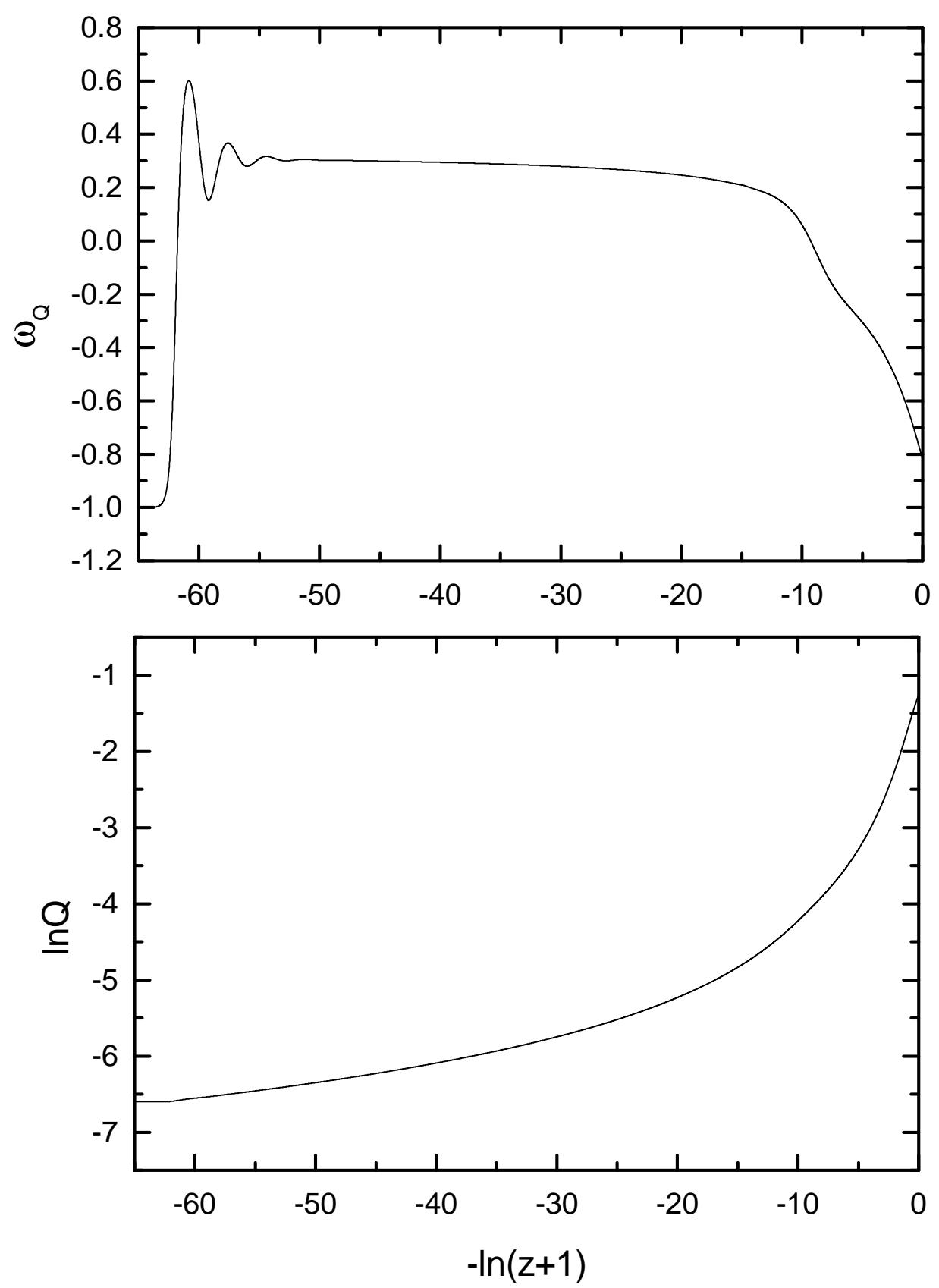

Fig. 4.- Evolution of the equation-of-state $\omega_{Q}$ and $Q$-field with redshift in the exponential form of inverse power-law model. We found that no limit on the tracking time can be obtained here. See the text for details. 


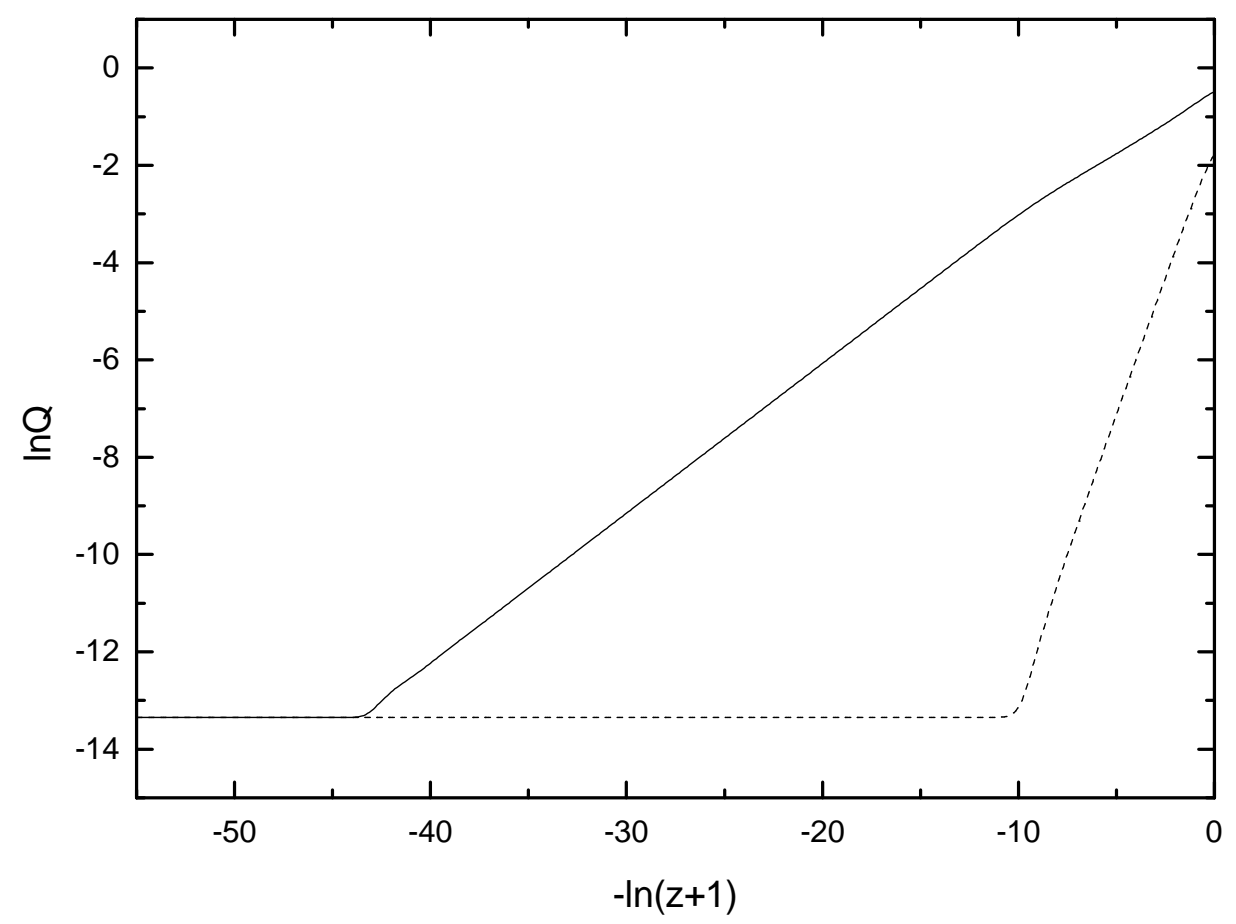

Fig. 5.- Evolution of the $Q$-field with redshift in both inverse power-law(dashed) and supergravity(solid) models. We take the initial condition $Q_{i}=H / 2 \pi$, where $H \sim 10^{-5}$, and find that the time the supergravity case enters the tracking regime is much earlier than that of the inverse power-law model. 